See discussions, stats, and author profiles for this publication at: https://www.researchgate.net/publication/345349660

\title{
Sleep Hygiene and Light Exposure Can Improve Performance Following Long- Haul Air Travel
}

Article in International Journal of Sports Physiology and Performance · November 2020

DOI: 10.1123/ijsp. 2019-0931

CITATION

1

9 authors, including:

Peter M Fowler

Queensland University of Technology

42 PUBLICATIONS 344 CITATIONS

SEE PROFILE

Q()

Charli Sargent

Central Queensland University

151 PUBLICATIONS 2,546 CITATIONS

SEE PROFILE

Some of the authors of this publication are also working on these related projects:

The Stay Healthy Project View project

Lower-Limb Sports Compression Garments Improve Exercise Performance and Muscle Blood Flow During Repeated-Sprint Cycling View project
READS

241

Heidi R Thornton

La Trobe University

SEE PROFILE

Amy E Mendham

South African Medical Research Council

37 PUBLICATIONS 271 CITATIONS

SEE PROFILE
52 PUBLICATIONS 570 CITATIONS 


\title{
Sleep Hygiene and Light Exposure Can Improve Performance Following Long-Haul Air Travel
}

\author{
Peter M. Fowler, Wade Knez, Heidi R. Thornton, Charli Sargent, Amy E. Mendham, \\ Stephen Crowcroft, Joanna Miller, Shona Halson, and Rob Duffield
}

\begin{abstract}
Purpose: To assess the efficacy of a combined light exposure and sleep hygiene intervention to improve team-sport performance following eastward long-haul transmeridian travel. Methods: Twenty physically trained males underwent testing at 09:00 and 17:00 hours local time on 4 consecutive days at home (baseline) and the first 4 days following 21 hours of air travel east across 8 time zones. In a randomized, matched-pairs design, participants traveled with (INT; $n=10)$ or without $(\mathrm{CON} ; \mathrm{n}=10)$ a light exposure and sleep hygiene intervention. Performance was assessed via countermovement jump, 20-m sprint, $T$ test, and Yo-Yo Intermittent Recovery Level 1 tests, together with perceptual measures of jet lag, fatigue, mood, and motivation. Sleep was measured using wrist activity monitors in conjunction with self-report diaries. Results: Magnitude-based inference and standardized effect-size analysis indicated there was a very likely improvement in the mean change in countermovement jump peak power (effect size $1.10, \pm 0.55)$, and likely improvement in $5-\mathrm{m}(0.54, \pm 0.67)$ and $20-\mathrm{m}(0.74, \pm 0.71)$ sprint time in INT compared with CON across the 4 days posttravel. Sleep duration was most likely greater in INT both during travel $(1.61, \pm 0.82)$ and across the 4 nights following travel $(1.28, \pm 0.58)$ compared with CON. Finally, perceived mood and motivation were likely worse $(0.73, \pm 0.88$ and $0.63, \pm 0.87)$ across the 4 days posttravel in CON compared with INT. Conclusions: Combined light exposure and sleep hygiene improved speed and power but not intermittent-sprint performance up to 96 hours following long-haul transmeridian travel. The reduction of sleep disruption during and following travel is a likely contributor to improved performance.
\end{abstract}

Keywords: soccer, jet lag, team sport, travel fatigue

The interrelated impact of travel-induced fatigue and jet lag symptoms can negatively affect cognitive and physical performance following long-haul transmeridian air travel and is of concern for athletic populations. ${ }^{1-3}$ Conditions encountered during travel, such as the uncomfortable seating arrangements, noise levels, and stopovers, may disrupt sleep and induce fatigue. ${ }^{2,3}$ Following travel, jet lag symptoms, particularly sleep disruption and daytime fatigue, result from a misalignment in timing between an individual's endogenous circadian system and the light-dark cycle at the destination. ${ }^{4-6}$ The circadian rhythms of numerous physiological and psychological variables, that typically have an early morning nadir and a late-afternoon peak, are expected to be misaligned with the new local time, and could result in worse performance ${ }^{4,7}$ Following arrival at the destination, the circadian system gradually aligns with the light-dark cycle of the new time

Fowler is with the Athlete Health and Performance Research Center, Aspetar Orthopedic and Sports Medicine Hospital, Doha, Qatar, and the School of Exercise and Nutrition Sciences, Queensland University of Technology, Brisbane, QLD, Australia. Knez is with the School of Human Movement and Nutrition Sciences, University of Queensland, Brisbane, QLD, Australia. Thornton is with the Gold Coast Suns Football Club, Metricon Stadium, Carrara, QLD, Australia. Sargent is with the Appleton Inst for Behavioural Science, Central Queensland University, Adelaide, SA, Australia. Mendham is with the Div of Exercise Science and Sports Medicine, University of Cape Town, Cape Town, South Africa. Crowcroft is with the New South Wales Inst of Sport, Performance Support, Physiology and Nutrition, Sydney, NSW, Australia. Miller is with Performance Services, Australian Institute of Sport, Canberra, ACT, Australia. Halson is with the School of Behavioural and Health Sciences, Australian Catholic University, McAuley at Banyo, QLD, Australia. Duffield is with the Sport and Exercise Discipline Group, University of Technology Sydney, Sydney, NSW, Australia. Fowler (pete@recoveryatthehub. com.au) is corresponding author. zone. Yet, until fully aligned, which takes approximately 0.5 and 1 day per time zone crossed west and east, respectively, these jet lag symptoms and performance reductions are likely to be present. ${ }^{5,8}$ Jet lag symptoms, particularly sleep disruption, have consistently been reported during and following simulated ${ }^{2,3}$ and actual ${ }^{1}$ longhaul transmeridian travel, and negatively affect ensuing physical performance, likely through increased physiological and psychological fatigue. ${ }^{1-3}$ As training and competition can occur within 24 to 48 hours after long-haul travel for elite team-sport athletes, practical and effective interventions are required to aid preparation upon arrival.

Several recent reviews have recommended the use of bright light and/or melatonin ingestion at targeted times pre, during, and posttravel to accelerate alignment of the circadian system to the new time zone and attenuate performance reductions. ${ }^{6,9}$ Indeed, under controlled laboratory conditions, combined light exposure and melatonin administration were more effective at inducing phase shifts in dim light melatonin onset compared with either in isolation. ${ }^{10}$ However, considering the potential unfavorable side effects of pharmacological interventions on performance, ${ }^{11,12}$ targeted light exposure is currently the most suitable method of accelerating phase shifts in athletes. ${ }^{6,9}$ Currently, there is limited data on the efficacy of bright light to enhance the recovery of physical performance in team-sport athletes following transmeridian air travel. ${ }^{13}$ This could be due to logistical commitments reducing light exposure time and/or difficulty in preventing incidental light exposure at inappropriate times. ${ }^{13}$

It is plausible that in addition to utilizing targeted light exposure to accelerate time zone adaptation, jet lag symptoms could be managed to facilitate daytime functioning. As sleep disruption is particularly evident both during ${ }^{2}$ and following ${ }^{1}$ long-haul transmeridian travel, it may be a central factor impacting 
performance, due to resultant physiological and psychological fatigue. ${ }^{1}$ Thus, sleep hygiene strategies that minimize sleep disruption during and after travel may enhance performance and recovery posttravel. Though these strategies are advocated to improve sleep, ${ }^{14}$ it is not known whether they are effective in minimizing sleep disruption during and following long-haul transmeridian travel, particularly under conditions of circadian misalignment. ${ }^{3}$

The aim of the present study was to assess the efficacy of a combined light exposure and sleep hygiene intervention applied during and following travel to reduce the impact of eastward longhaul transmeridian travel on sleep, subjective jet lag and fatigue, and physical performance. It was hypothesized that the intervention would be effective at reducing the impact of travel on sleep and performance.

\section{Methods}

\section{Participants}

Twenty healthy, physically trained males were recruited to participate in the study (mean [[SD]; age 21.9 [3.6] y, height 178.3 [6.5] cm, and body mass $77.1[7.67] \mathrm{kg}$ ). All participants had recent training history in a range of athletic events, including running, football, rugby, and soccer and were involved in physical training (aerobic and strength) at least 2 to 3 times per week. Prior to the commencement of the study, participants were informed of any associated risks and provided verbal and written informed consent. The study was approved by the Anti-Doping Lab Qatar Institutional Ethics Review Board (EXT2014000003).

\section{Experimental Design}

The full experimental design for the present study has been previously described, including a full description of all experimental procedures for physical performance (countermovement jump [CMJ] test, 20-m sprint test, the $T$ test, ${ }^{15}$ and Yo-Yo Intermittent Recovery Level 1 test) and sleep and perceptual (Liverpool John Moore's University jet lag questionnaire, Brunel Mood Scale, ${ }^{16}$ session of rating of perceived exertion, and physical feeling) measures. ${ }^{1}$ The current study reports the efficacy of a practical travel intervention on team-sport performance for the eastbound component of the aforementioned study (Figure 1). Participants completed a minimum of 2 familiarization sessions with all experimental procedures. Baseline performance was assessed at

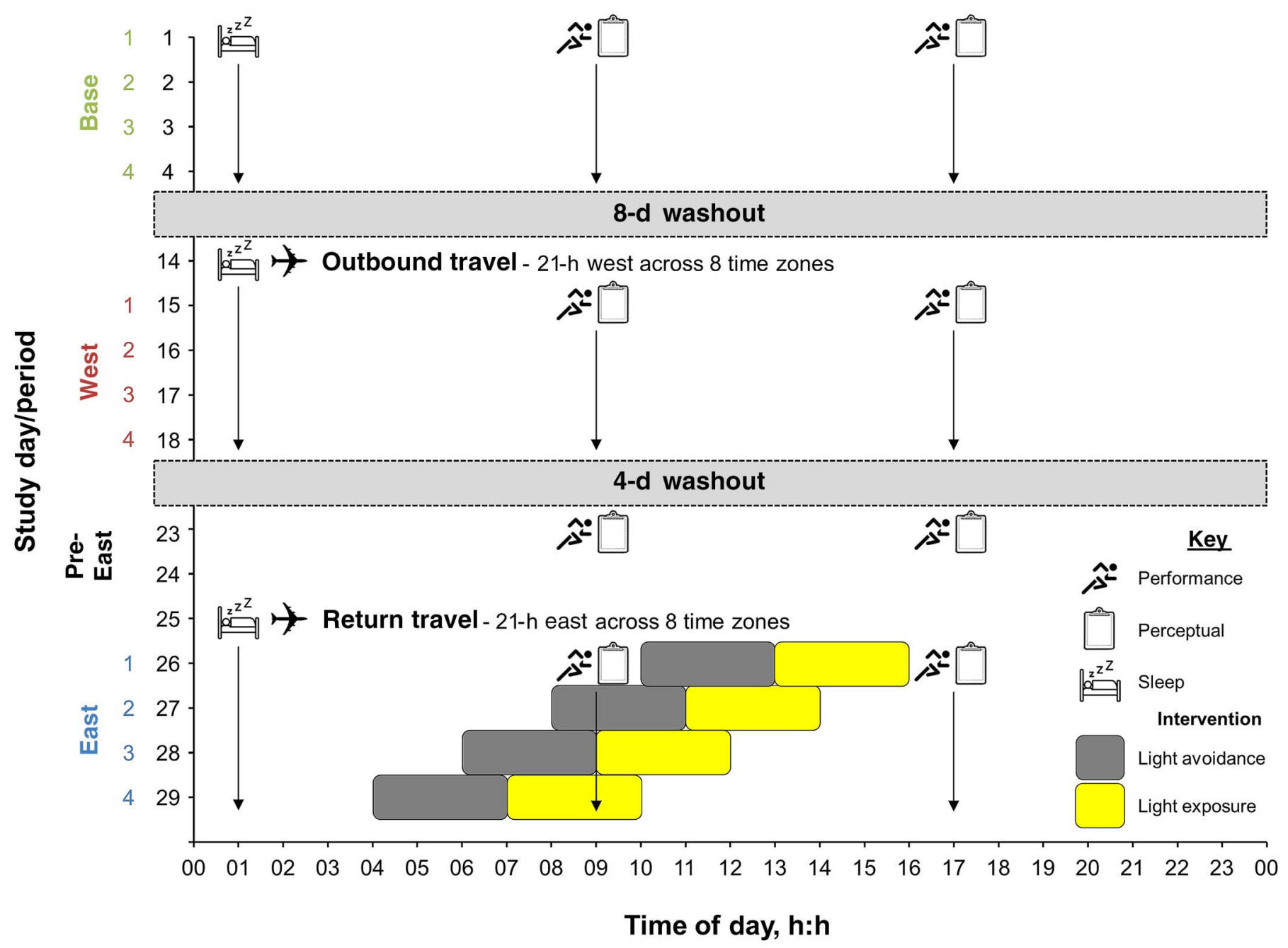

Figure 1 - Schematic outline of the study design including timeline of performance, perceptual, and sleep data collection, together with the light exposure and avoidance schedule for the intervention group. During each washout, participants carried out "normal" daily activities, with strenuous activity avoided 24 hours before each data-collection period. 
09:00 AM and 17:00 PM local time (deemed relevant to training and competition) on 4 consecutive days prior to any travel. In a randomized matched-pairs design, participants were matched based on their age and baseline physical performance level before independent randomization to an intervention (INT) or control (CON) group for the duration of the study. The intervention group received a combined light exposure and sleep hygiene intervention; the control group was not provided with any instruction other than to maintain normal behavior. Data were collected at the same time of day (local time) on the first 4 days following travel from Qatar to Australia.

Sleep was measured via actigraphy (Actiwatch-64; Philips Respironics, Bend, OR) throughout the aforementioned data collection periods and during travel, and perceptual data were collected immediately prior to all performance testing sessions. During baseline and following travel, participants slept at home. Participants abstained from caffeine, alcohol, and additional strenuous activity in the 24 hours prior to, and during, each data collection period. Food and fluid intake were documented throughout the data collection periods in a food diary, with participants instructed to replicate their recorded intake during baseline as closely as possible following travel. Participants were provided with a standardized 1.5 to $2.0 \mathrm{~g} / \mathrm{kg}$ body mass of carbohydrate, including $600 \mathrm{~mL}$ of fluid (Gatorade ${ }^{\mathrm{TM}}$, Chicago, IL) immediately following all performance testing sessions. Prior to all performance testing, urine specific gravity was assessed (Digital Refractometer, Atago, WA) to determine hydration status from a midstream urine sample.

\section{Travel}

Participants travelled in economy class for all flights. The departure and arrival times were 18:25 hours Arabia Standard Time and 22:20 hours the next day Australian Eastern Standard Time (Arabia Standard Time $+8 \mathrm{~h}$ ). In total, there were 3 flights, with 21 hours of travel ( $17 \mathrm{~h}$ total flight duration and $4 \mathrm{~h}$ total transit time) across 8 time zones:

1. Doha, Qatar (18:25 h local time) to Abu Dhabi, United Arab Emirates (20:20 h)

2. Abu Dhabi, United Arab Emirates (22:20 h) to Perth, Australia $(13: 50 \mathrm{~h}+1 \mathrm{~d})$

3. Perth, Australia (16:00 h) to Sydney, Australia (22:20 h)

\section{Interventions}

Sleep Hygiene During Travel. Participants in the INT group were provided with ear plugs (Mack's Pillow Soft ${ }^{\circledR}$ Silicone Putty Earplugs; McKeon Products Inc, Warren, MI), noise-cancelling headphones (Sony MDR-10RNC; Sony, Tokyo, Japan), a sleep mask (Sweet Dreams Eye Mask; Dream Essentials, Paradise Point, Queensland, Australia), and a neck pillow (Traveler's Pillow; Therapeutic Pillow International, Cheltenham, Victoria, Australia). Participants were instructed to utilize these products to maximize sleep from 23:30 to 08:30 hours Gulf Standard Time on Flight 2. Thus, participants were encouraged to sleep when it was night time in the city of departure (ie, during biological night time when the circadian system is promoting sleep). Furthermore, in the hour prior to attempting to sleep, participants were instructed to restrict computer, TV, and phone use. These specific interventions were based on evidence that exposure to light and noise can reduce sleep quality and aimed to increase comfort and induce the physiological state required for sleep onset, without pharmacological aids. ${ }^{14}$ Finally, to ensure participants in the INT group could maximize their sleep in the aforementioned "sleep window," they were instructed to avoid the evening meal on the plane and instead ate at the airport prior to departure. While no instructions on activity levels outside of the "sleep window" were provided to the INT group, the CON group was not provided with any instructions regarding behavior during travel (ie, sleep, activity, or eating patterns). Participants were seated in various places on the plane, but individuals in different groups were not seated together.

Light Exposure and Sleep Hygiene Following Travel. The INT group's exposure to, and avoidance of, light was controlled following arrival. Specifically, participants were instructed and supervised to seek natural outdoor light or utilize wraparound sunglasses to minimize exposure to light (Contractor Smoke Safety Glasses; DeWalt, Melbourne, Australia) at specific times (Figure 1). This pattern of light exposure/avoidance provides an "advance" signal and facilitates the adjustment of the circadian system so that it is aligned with the new time zone. ${ }^{17-19}$ If natural outdoor light was not available at these specific times (ie, participants were indoors), artificial bright light emitting glasses (Re-Timer ${ }^{\mathrm{M}}$, Bedford Park, Australia) were used instead. These glasses provide 506 lux of blue-green 500-nm dominant wavelength, ultraviolet-free light at $12 \mathrm{~mm}$ and have previously been reported to induce phase shifts of circadian rhythm. ${ }^{19}$ Sleep hygiene recommendations ${ }^{14,17}$ were also provided to participants in the INT group. Specifically, in the hour prior to bed, participants were instructed to limit computer, TV, and phone use and to dim their bedroom lights. Cool, quiet, and dark conditions were ensured throughout the sleep period, utilizing ear plugs and sleep masks if necessary. Participants slept in their own room at home. The INT group were instructed and supervised to increase their time in bed compared with normal by going to bed earlier. In contrast, the participants in the CON group were not provided with the aforementioned instructions other than to maintain normal behavior.

\section{Statistical Analysis}

Normality of the observed data was initially assessed using quantilequantile (Q-Q) plots and was deemed plausible in all instances. As reported elsewhere, ${ }^{1}$ no significant $(P<.05)$ differences were observed for any measures between baseline day 1 and pretravel eastwards day 1; therefore, the baseline was considered appropriate for all posttravel comparisons. The raw change in all performance variables on each of the 4 days following travel were calculated from the corresponding day at baseline (ie, posttravel day 1 was compared with baseline day 1). Raw values for each of the 4 days at baseline and following travel were used for all perceptual variables. Raw values for 3 nights at baseline (days 1-3) and the first 4 nights following travel (day of arrival and days 1-3) were used for all sleep variables. Differences between condition (INT and CON) within each study period (baseline and posttravel), and between study periods within each condition were analyzed using linear mixed models. This type of analysis is preferred, as it allows for missing data and can model between-subject variability. ${ }^{20}$ Specifically, in these models, an unstructured covariance structure was used, using the lme4 package in R Studio statistical software (version 0.99.446, Boston, MA). Athlete identification was included as the random effect in the models, and day or condition was included as fixed effects, either independently, or as an interaction. Following this, the least squares mean test was used to identify the mean difference in the outcome variable depending on the comparison being made.

Furthermore, standardized effect sizes were calculated as previously described ${ }^{21}$ using the $P$ value and $t$ statistic provided 
from the linear mixed models categorized using the thresholds of $<0.2$ trivial, 0.21 to 0.60 small, 0.61 to 1.20 moderate, 1.21 to 2.0 large, and $>2.0$ very large. ${ }^{22}$ Furthermore, a magnitude-based approach was adopted, ${ }^{22}$ where differences were considered real if there was a $>75 \%$ likelihood of exceeding the smallest worthwhile difference (0.20) and are described as: $75 \%$ to $95 \%$, likely; $95 \%$ to $99.5 \%$, very likely; and $99.5 \%$, most likely. ${ }^{21}$ Descriptive statistics are presented as mean (SD), whereas all other data are reported as ES, $\pm 90 \%$ confidence limits, unless otherwise stated.

\section{Results}

\section{Performance}

At both 09:00 and 17:00 hours, INT had a very likely $(1.10, \pm 0.55)$ and likely $(0.70, \pm 0.71)$ beneficial effect on the mean change across the 4 days in CMJ peak power and height, respectively, compared with CON (Figure 2). At 17:00 hours, INT had a likely beneficial effect on mean change across the 4 days in $5-\mathrm{m}(0.54, \pm 0.67)$ and 20 -m $(0.74, \pm 0.71)$ sprint time compared with CON (Figure 2). No substantial effects $(\mathrm{ES}<0.20)$ between conditions were observed for the mean change in $T$-test time or Yo-Yo Intermittent Recovery Level 1 test distance (Figure 3).

Morning (AM) Testing (09:00 h Local Time). INT had a very likely beneficial effect on the change in $\mathrm{CMJ}$ peak power on days 2 $(0.87, \pm 0.54)$ and $4(0.51, \pm 0.54)$, respectively, compared with CON (Figure 2). INT also had a likely $(0.63, \pm 0.65)$ beneficial effect on the change in CMJ height on day 3 compared with CON.

Afternoon (PM) Testing (17:00 h Local Time). INT had a likely beneficial effect on the change in CMJ peak power on days 1 (0.41, $\pm 0.46)$ and $3(0.46, \pm 0.45)$, and a most likely beneficial effect on day $2(1.01, \pm 0.46)$ compared with CON (Figure 2$)$. INT also had a likely beneficial effect on the change in CMJ height on days 1 to 3 $(0.52, \pm 0.60 ; 0.59, \pm 0.60$; and $0.51, \pm 0.56)$ compared with CON. INT had a likely beneficial effect on the change in 5-m sprint time on days 2 to $4(0.51, \pm 0.59 ; 0.66, \pm 0.56$; and $0.60, \pm 0.57)$ and the change in 20 -m sprint time on all 4 days $(0.48, \pm 0.67 ; 0.72, \pm 0.67$; $0.49, \pm 0.63$; and $0.53, \pm 0.64$ ) compared with CON (Figure 2).

\section{Sleep}

During travel, sleep duration was most likely greater $(1.61, \pm 0.82)$ in INT compared with CON (06:18 [01:00] vs 03:54 [01:36] h). However, no substantial differences in sleep efficiency $(\mathrm{ES}<0.20)$ were observed between INT and CON (79.8\% [6.5\%] vs 73.8\% [16.2\%]) during travel. Across the 3 nights following travel, mean time in bed, sleep duration, and sleep efficiency was very likely, most likely, and likely greater $(1.28, \pm 0.58 ; 0.99, \pm 0.55$; and $0.64, \pm 0.54)$, whereas mean sleep onset was very likely earlier $(0.95, \pm 0.61)$ in INT compared with CON (Table 1). Specifically, on nights 1 and 2 , time in bed was likely greater $(0.58, \pm 0.48$ and $0.54, \pm 0.49)$ and sleep onset was likely earlier $(0.53, \pm 0.54$ and $0.72, \pm 0.56)$ in INT compared with CON. Sleep duration was likely greater on nights 1 and $3(0.58, \pm 0.51$ and $0.43, \pm 0.55)$, and very likely greater on night 2 in INT compared with $\mathrm{CON}(0.78, \pm 0.53)$.

\section{Perceptual}

Mean motivation was likely reduced at 09:00 hours $(0.64, \pm 0.87)$ and 17:00 hours $(0.63, \pm 0.87)$, and mean mood was likely worse $(0.73, \pm 0.88)$ across the 4 days following travel in CON compared with INT. No substantial differences $(\mathrm{ES}<0.20)$ between conditions were observed for subjective jet lag ratings (Table 2).
Morning (AM) Testing (09:00 h Local Time). Sleep symptoms were likely better $(0.64, \pm 0.49)$ on day 1 , but motivation was likely worse $(0.58, \pm 0.73)$ on days 3 and 4 in CON compared with INT.

Afternoon (PM) Testing (17:00 h Local Time). Function, mood, and motivation were all likely worse $(0.42, \pm 0.45 ; 0.45, \pm 0.49$; and $0.68, \pm 0.53)$ on day 3 in CON compared with INT.

\section{Discussion}

Results from the present study suggest that a combined light exposure and sleep hygiene intervention reduced sleep disruption during and following travel, improved perceived mood and motivation, and ultimately enhanced posttravel lower body power. These improvements have implications for any training or competition requirements within 48 to 72 hours following long-haul travel.

The combined intervention improved CMJ performance compared with the control condition up to 4 days posttravel, with the largest difference between conditions noted on day 2. In addition, 5- and 20-m sprint times were faster in the intervention group on all 4 days posttravel, with performance returning to baseline earlier (72 h) in the intervention compared with the control ( $96 \mathrm{~h}$ ) group. Accordingly, the intervention was successful in facilitating adjustment to the new time zone; however, in the absence of a measure of circadian adaptation it is not possible to confirm this assumption. It is also possible that the acute effect of light exposure on alertness and/or the improvement in sleep, perceived mood and motivation, ${ }^{6,13,24}$ independent of any circadian adaptation, resulted in performance improvements. Regardless, the results of the present study show combining light and sleep hygiene can limit the reduction in lower body power typically observed following transmeridian travel. , $^{1,4,5}$

Negligible effects of a similar light exposure intervention were observed on daily changes in grip-strength performance and body temperature in the 4 days following long-haul travel east across 5 time zones in elite female soccer players. ${ }^{13}$ One potential explanation for the lack of efficacy is the difficulty encountered when implementing such interventions in the field with elite athletes. ${ }^{25}$ On some occasions, the duration of light exposure was reduced to accommodate training, whereas on other occasions, it was difficult to prevent incidental light exposure at the incorrect times for inducing a circadian phase advance. ${ }^{13,25}$ In the present study, exposure to, and avoidance of, bright light at appropriate times was tightly controlled and may have contributed to the faster performance adjustment to the new time zone.

Previous research highlights sleep disruption, together with greater perceived fatigue and reduced motivation, as a reason for reduced performance following long-haul travel. ${ }^{1,2}$ Therefore, the greater sleep duration and reduced perceived fatigue evident in the intervention group could also explain the noted improvements in lower body power, despite no effect on intermittent-sprint capacity. Previous evidence suggests that individuals are able to overcome the effects of sleep loss to complete short-duration/explosive exercise, yet are unable to maintain performance in sustained or repeated exercise bouts, probably due to reduced motivation to maintain high-intensity performance. ${ }^{26}$ Thus, the level of improvement in sleep during and after travel in the intervention group may have not been large enough to prevent the reductions observed in prolonged intermittent-sprint performance.

One of the main causes of travel-induced fatigue and performance reductions following arrival can be sleep loss during prolonged flights that span a night time sleep episode. ${ }^{1,2}$ Indeed, mean sleep durations of $\sim 3$ hours have been reported during both simulated $^{2,3}$ and actual $^{1}$ long-haul transmeridian travel due to the 

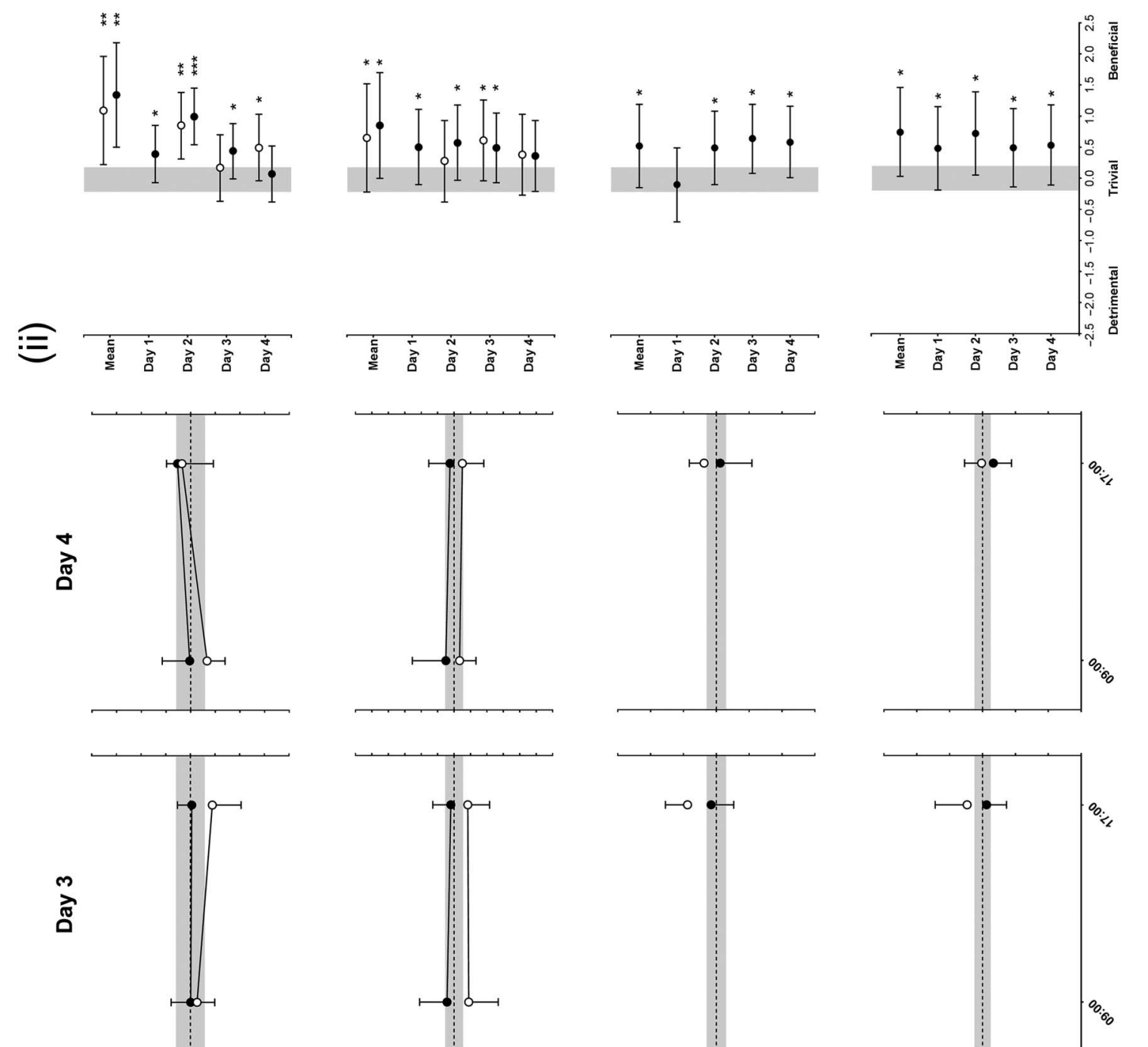

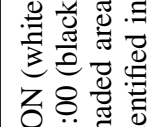

8 运

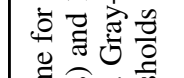

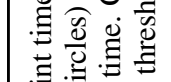

楁司导

हैㅇํำ:

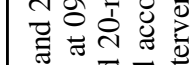

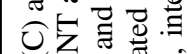

实包言完

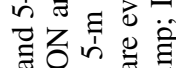

คิธ

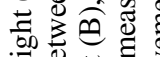

区

휴월

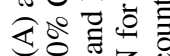

过全号官

造记

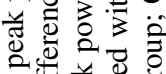
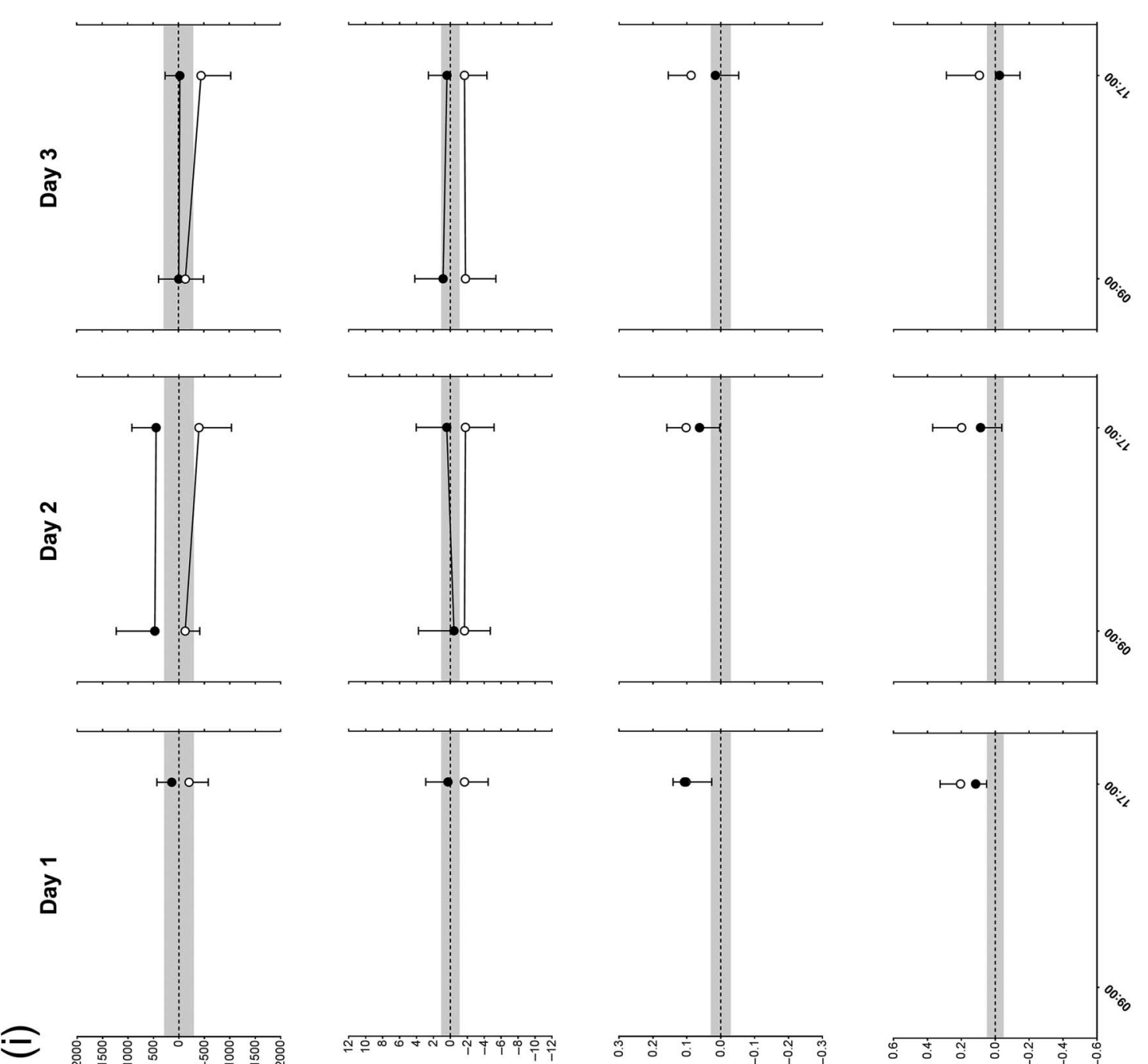

Є

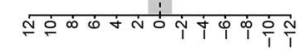
$\varangle \quad M$ 'а мемоd yeəd $\nabla$

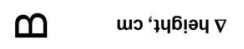

0

s'əu!n แ-s $\nabla$

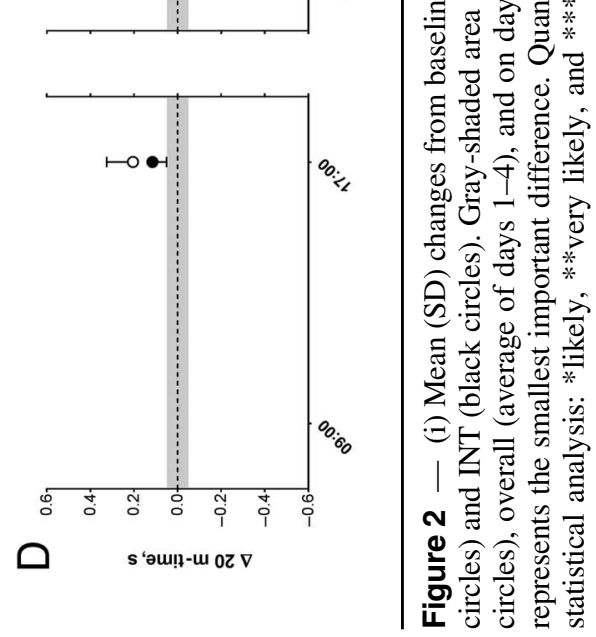



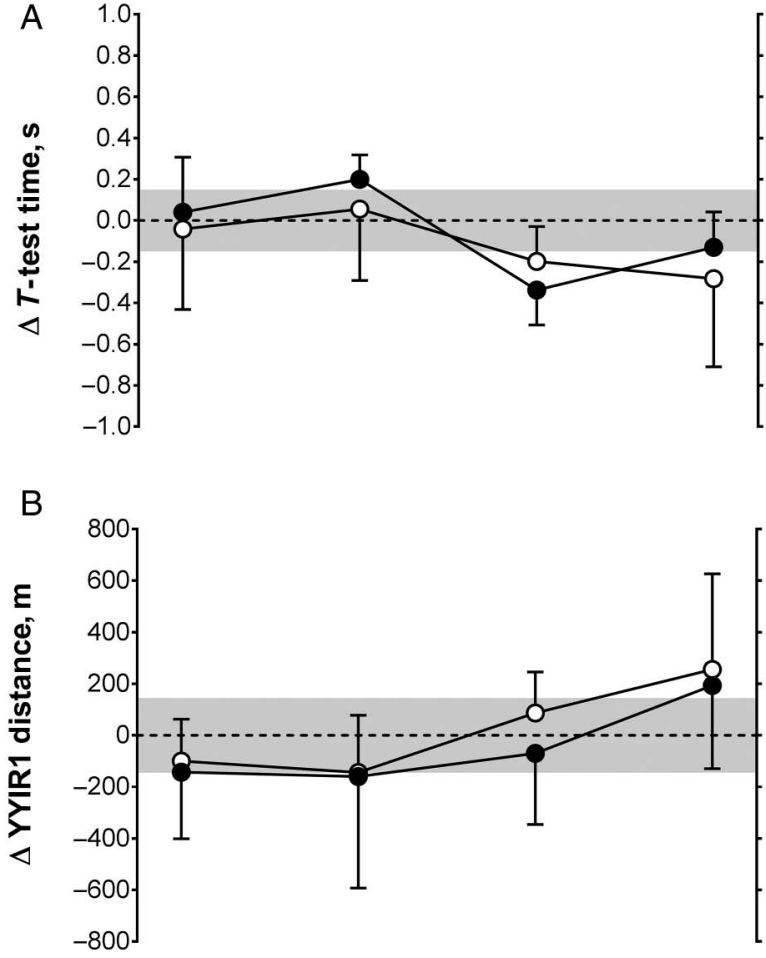

C
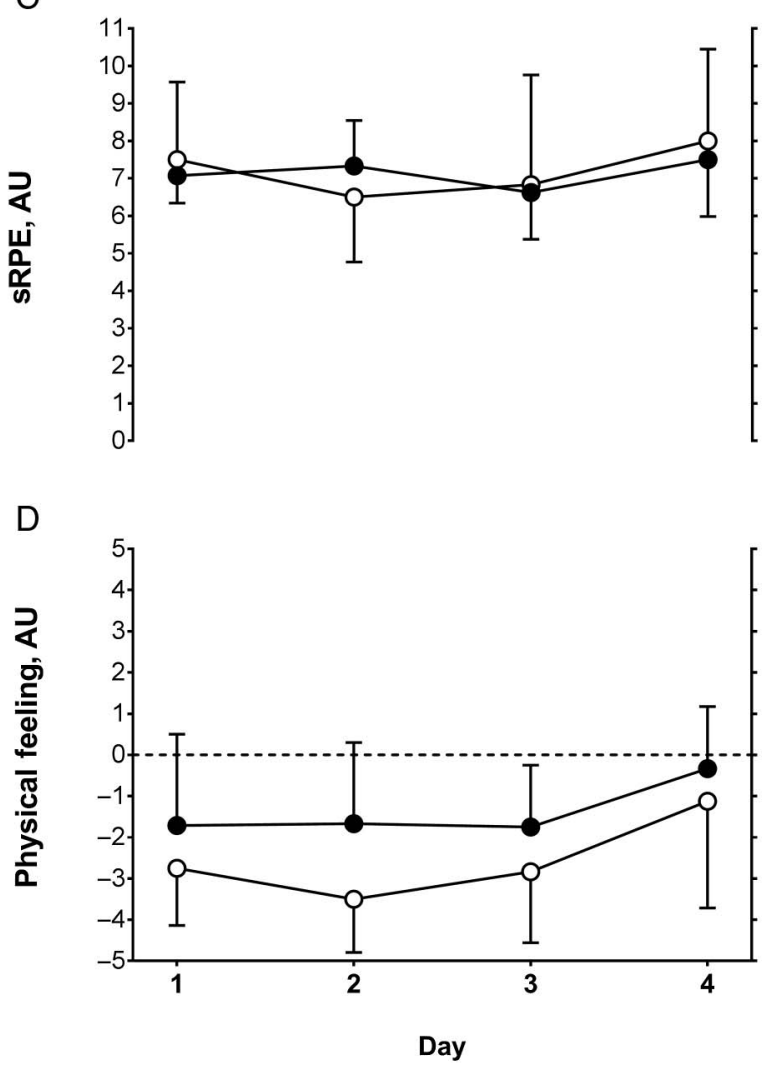
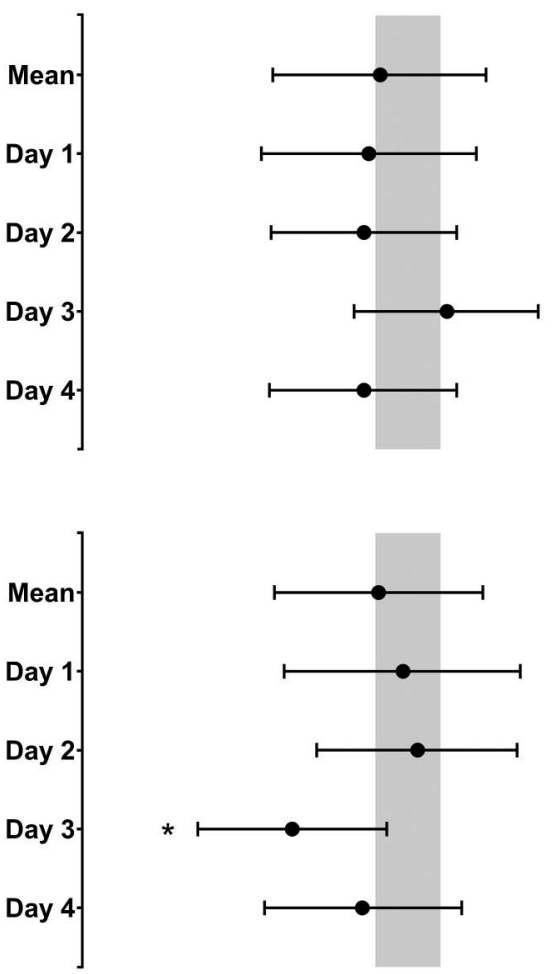

Mean-
Day 1
Day 2
Day 3
Day 4
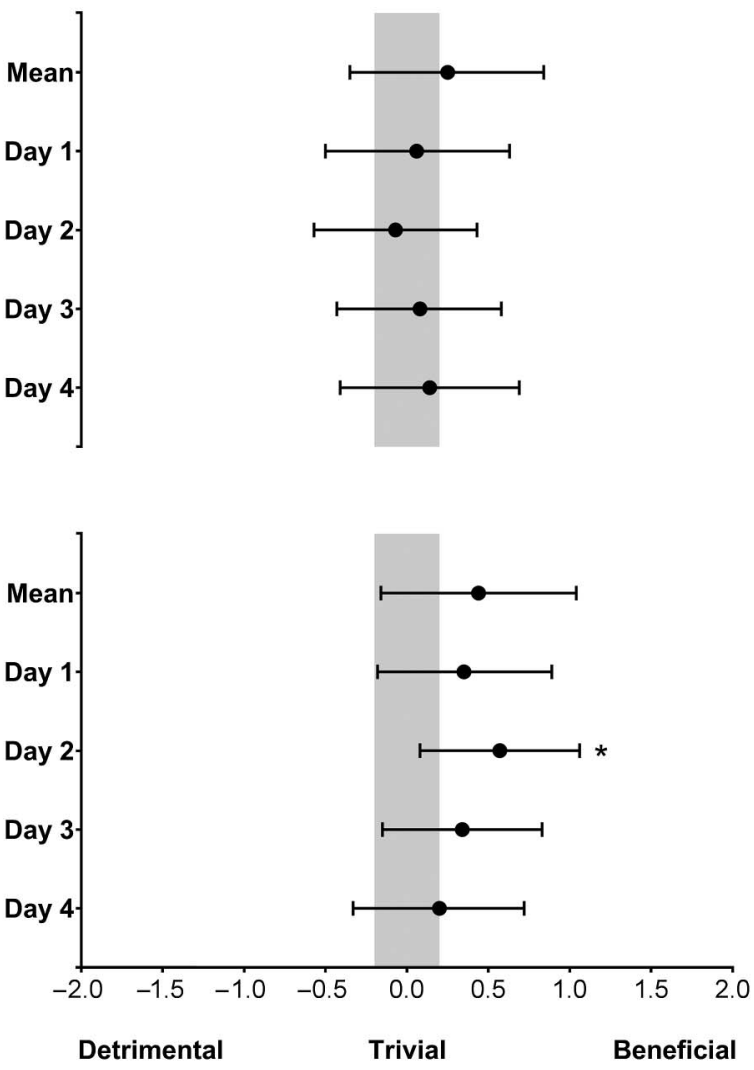

Figure 3 - Left column: Mean (SD) changes from baseline in agility (A) and YYIR1 (B) performance, mean (SD) sRPE (C), and physical feeling (D) on days 1 to 4 posttravel at 17:00 local time for CON (white circles) and INT (black circles). Gray-shaded area indicates the typical error of each measure. Right column: Standardized differences ( $\pm 90 \%$ CIs) between CON and INT at 17:00, overall (average of days 1-4) and days 1 to 4 posttravel for mean change from baseline in agility (A) and YYIR1 (B) performance, mean sRPE (C), and physical feeling (D). Gray-shaded area represents the smallest important difference. Quantitative chances of INT being beneficial or detrimental compared with CON for each measure are evaluated according to the thresholds identified in statistical analysis: *likely, **very likely, and ***most likely. CI indicates confidence interval; CON, control group; INT, intervention group; sRPE, session of rating of perceived exertion; YYIR1, Yo-Yo Intermittent Recovery Level 1 test. 
Table 1 Sleep Patterns, Quantity, and Quality During Baseline (BASE) and Following Travel (EAST) for INT and CON

\begin{tabular}{|c|c|c|c|c|c|c|}
\hline & & Arrival & 1 & 2 & 3 & Mean (days 1-3) \\
\hline \multicolumn{7}{|c|}{ Time in bed, h:min } \\
\hline \multirow[t]{2}{*}{ BASE } & $\mathrm{CON}$ & & 07:13 (01:06) & $07: 23(01: 22)$ & 07:21 (00:58) & 07:19 (01:03) \\
\hline & INT & & $07: 44(00: 58)$ & $07: 27(00: 54)$ & $07: 44(00: 35)$ & 07:38 (00:40) \\
\hline \multirow[t]{2}{*}{ EAST } & $\mathrm{CON}$ & 07:20 (00:43) & $06: 04(01: 29)^{\mathrm{c}, \mathrm{e}}$ & $06: 11(01: 52)^{\mathrm{c}, \mathrm{e}}$ & $06: 24(01: 22)^{\mathrm{e}}$ & $06: 04(01: 22)^{\mathrm{b}, \mathrm{e}}$ \\
\hline & INT & 07:05 (01:43) & $07: 13(00: 53)^{\mathrm{f}}$ & $07: 41(02: 30)$ & $07: 09(01: 20)^{\mathrm{e}}$ & 07:27 (01:24) \\
\hline \multicolumn{7}{|c|}{ Sleep onset, hh:mm } \\
\hline \multirow[t]{2}{*}{ BASE } & $\mathrm{CON}$ & & 23:20 (01:07) & 23:11 (01:20) & 23:04 (01:10) & 23:12 (01:09) \\
\hline & INT & & 23:01 (01:00) & $23: 14(00: 50)$ & $22: 56(00: 42)$ & 23:04 (00:40) \\
\hline \multirow[t]{2}{*}{ EAST } & $\mathrm{CON}$ & 01:07 (01:14) & $00: 22(01: 24)^{\mathrm{c}, \mathrm{e}}$ & $00: 27(01: 46)^{\mathrm{c}, \mathrm{e}}$ & $23: 54(01: 41)^{\mathrm{e}}$ & $00: 21(01: 25)^{\mathrm{b}, \mathrm{e}}$ \\
\hline & INT & 01:21 (01:19) & $23: 37(00: 59)^{\mathrm{f}}$ & $22: 52(02: 01)$ & $23: 36(00: 38)^{f}$ & $23: 15(01: 14)$ \\
\hline \multicolumn{7}{|c|}{ Sleep offset, hh:mm } \\
\hline \multirow[t]{2}{*}{ BASE } & $\mathrm{CON}$ & & $06: 26(00: 28)$ & $06: 20(00: 28)$ & 06:09 (00:38) & $06: 19(00: 28)$ \\
\hline & INT & & $06: 29(00: 36)$ & $06: 22(00: 22)$ & $06: 25(00: 26)$ & $06: 25(00: 25)$ \\
\hline \multirow[t]{2}{*}{ EAST } & $\mathrm{CON}$ & 08:25 (01:20) & 06:25 (00:30) & $06: 35(00: 30)^{\mathrm{f}}$ & $06: 14(00: 45)$ & $06: 23(00: 32)$ \\
\hline & INT & $08: 25(01: 22)$ & $06: 50(00: 29)^{\mathrm{f}}$ & 06:32 (00:50) & $06: 42(01: 12)$ & $06: 41(00: 33)$ \\
\hline \multicolumn{7}{|c|}{ Sleep duration, h:min } \\
\hline \multirow[t]{2}{*}{ BASE } & $\mathrm{CON}$ & & $06: 25(01: 10)$ & 06:12 (01:19) & $06: 21(00: 54)$ & $06: 19(01: 03)$ \\
\hline & INT & & $06: 49(00: 49)$ & $06: 30(00: 47)$ & $06: 50(00: 23)$ & $06: 43(00: 32)$ \\
\hline \multirow[t]{2}{*}{ EAST } & $\mathrm{CON}$ & $06: 30(00: 52)$ & $05: 14(01: 19)^{\mathrm{b}, \mathrm{d}}$ & $05: 16(01: 45)^{\mathrm{b}, \mathrm{f}}$ & $05: 36(01: 23)^{\mathrm{c}, \mathrm{e}}$ & $05: 15(01: 18)^{\mathrm{a}, \mathrm{e}}$ \\
\hline & INT & $06: 19(01: 43)$ & $06: 20(00: 47)^{\mathrm{f}}$ & 07:01 (02:19) & $06: 36(01: 16)$ & $06: 45(01: 17)$ \\
\hline \multicolumn{7}{|c|}{ Sleep efficiency, \% } \\
\hline \multirow[t]{2}{*}{ BASE } & $\mathrm{CON}$ & & $90.2(3.3)^{\mathrm{c}}$ & $86.5(2.8)^{\mathrm{c}}$ & $89.6(3.2)$ & $88.8(2.6)^{\mathrm{c}}$ \\
\hline & INT & & $91.4(3.5)$ & $91.3(5.0)$ & $91.5(2.0)$ & $91.4(3.3)$ \\
\hline \multirow[t]{2}{*}{ EAST } & $\mathrm{CON}$ & $88.8(5.8)$ & $86.5(3.4)^{\mathrm{e}}$ & $86.4(10.6)$ & $88.3(3.4)^{\mathrm{f}}$ & $87.2(5.2)^{\mathrm{c}}$ \\
\hline & INT & $89.2(2.7)$ & $88.1(4.4)^{\mathrm{f}}$ & $91.2(3.2)$ & $92.9(1.1)$ & $90.9(1.2)$ \\
\hline \multicolumn{7}{|c|}{ Subjective sleep quality, AU } \\
\hline \multirow[t]{2}{*}{ BASE } & $\mathrm{CON}$ & & $2.6(4.3)$ & $1.8(4.6)$ & $3.4(4.8)^{\mathrm{c}}$ & $2.9(4.3)$ \\
\hline & INT & & $1.3(2.6)$ & $1.5(1.6)$ & $0.4(2.4)$ & $1.4(2.3)$ \\
\hline \multirow[t]{2}{*}{ EAST } & $\mathrm{CON}$ & $3.8(6.4)^{\mathrm{c}}$ & $4.9(5.3)$ & $2.7(7.8)$ & $1.2(3.8)$ & $2.9(5.9)$ \\
\hline & INT & $10.2(8.6)^{\mathrm{d}}$ & $6.6(8.3)^{\mathrm{e}}$ & $2.0(3.9)$ & $-0.3(3.3)$ & $2.8(6.1)^{\mathrm{e}}$ \\
\hline
\end{tabular}

Abbreviations: CON, control group; INT, intervention group. Note: Time in bed (h:min): the period between going to bed and getting up. Sleep onset (hh:mm): the time at which a participant first fell asleep after going to bed. Sleep offset (hh:mm): the time at which a participant last woke before getting up. Sleep duration (h:min): the amount of time spent in bed asleep. Sleep efficiency (\%): sleep duration expressed as a percentage of time in bed. Subjective sleep quality (AU): These ratings were taken from the responses from the "sleep" subscale of the Liverpool John Moore's University jet lag questionnaire ${ }^{23}$; whereby there are 5 questions related to sleep quality in comparison with normal. Answers from each question are then pooled for summation into an overall sleep quality rating, with a greater overall value indicating worse sleep quality. Data are presented as mean (SD).

${ }^{a}$ Most likely different to INT. ${ }^{b}$ Very likely different to INT. ${ }^{c}$ Likely different to INT. ${ }^{\mathrm{d}}$ Almost certainly different to baseline. ${ }^{\mathrm{e}}$ Very likely different to baseline. ${ }^{\mathrm{f}}$ Likely different to baseline.

schedule, uncomfortable sleeping position, and/or cabin conditions. The sleep hygiene "devices" (ear plugs, eye masks, and neck pillows) and guidelines (electronic device use restrictions), together with providing specific advice on when to sleep, increased mean sleep duration during travel by 2.5 hours. Previous recommendations suggest that sleep during a flight should be scheduled to coincide with night time in the destination city to prevent the sleep/wake cycle from "anchoring" to the departure time zone. ${ }^{17,24}$ However, in the present study, participants were instructed to schedule sleep according to when it was night time in the departure city. This approach not only reduced sleep disruption during travel, but also did not have any negative consequences on sleep or subjective jet lag symptoms posttravel. However, further investigations regarding the specific timing of sleep during travel and its subsequent impact on posttravel outcomes are required.

A common symptom of jet lag following long-haul travel east is sleep disruption due to delayed sleep onset. ${ }^{1,27}$ In the present study, the intervention was effective at preventing the reduction in sleep duration observed posttravel in the control group, through increased time in bed and an earlier sleep onset, particularly on nights 1 and 2. Indeed, one of the specific sleep hygiene guidelines was to "increase time in bed compared with normal by going to bed earlier." Despite this, sleep durations were still relatively short (5$7 \mathrm{~h}$ ) in both groups. This is likely a result of having to get up early for the morning testing, which mirrors the requirements for players contracted to teams located in large cities, who are required to get 


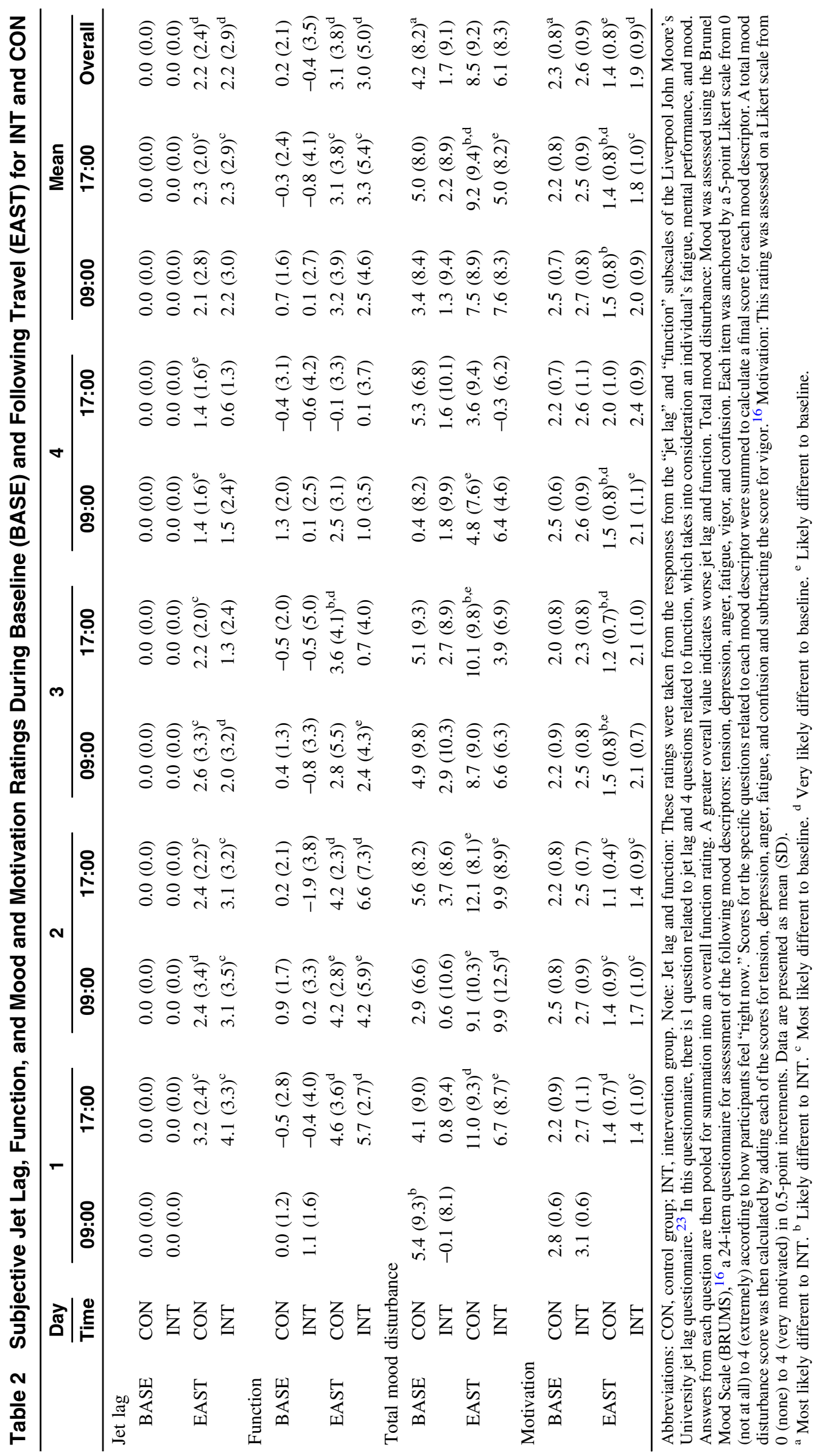


up at similar times to attend morning training. A similar acute sleep hygiene intervention increased sleep duration during, and following, 24 hours of simulated air travel in physically trained males. ${ }^{3}$ Although adherence to the prescribed interventions in INT was difficult to record, participants verbally confirmed regular use. However, the impact of chronotype was not assessed, which is recognized as a potential limitation. ${ }^{28}$ Moreover, while no differences in sleep quality were observed between the groups, subtle yet important differences may have been detected through polysomnography, which was unavailable in the present study. Regardless, these results suggest that sleep hygiene guidelines may be effective at reducing sleep disruption following travel.

Inducing phase shifts to accelerate the alignment of the circadian system with external day-night cycles can theoretically help to minimize the symptoms of jet lag. ${ }^{17}$ In the absence of a measure of phase shift in the present study, subjective symptoms of jet lag were used as a "proxy" for measuring circadian adaptation. ${ }^{23}$ It was hypothesized that the greatest differences in subjective jet lag between the groups would occur on day 4-as theoretically, by day 4 , the participants in the intervention group should be more adapted to the new time zone compared with those in the control group. However, comparable with the findings of Thompson et al, ${ }^{13}$ who performed a similar study with elite female soccer players, there were no differences in subjective jet lag ratings between the intervention and control conditions on day 4. Instead, mean mood and motivation across the 4 days were improved at 17:00 hours (local time), with function (fatigue, alertness, and mood) and motivation better on day 3 . These differences align with the greater sleep duration observed in the intervention group for the first 2 nights and given previous observations that sleep loss itself can exacerbate subjective ratings of fatigue, mood, and motivation, ${ }^{1,2,29}$ it may be that sleep interventions are worthy of further exploration for combating the negative effects of transmeridian travel.

\section{Practical Applications}

Given the negative impact of both the demands of travel itself and the ensuing jet lag upon arrival, unless appropriate travel strategies are implemented, then athlete health, well-being, and performance are likely to suffer. The present study provides evidence to suggest that practitioners should use sleep hygiene and light exposure following travel to reduce the impact of long-haul transmeridian travel on athlete sleep, mood, and performance. These guidelines will assist athlete's preparedness for both training and competition following arduous international travel.

\section{Conclusions}

The present study demonstrates the efficacy of a practical intervention for reducing sleep disruption during, and following, longhaul transmeridian travel; together with enhancing mood, and performance for up to 96 hours postarrival. Collectively, this study provides coaches and practitioners with an evidence-based, practical strategy for aiding athlete arrival following transmeridian travel and ensuing preparation for training and competition.

\section{Acknowledgments}

The authors would like to thank the participants for their time and enthusiasm, and gratefully acknowledge the allocation of resources provided by the Aspire Zone Foundation, Aspetar Orthopaedic and Sports Medicine Hospital, and University of Technology Sydney in support of this project.

\section{References}

1. Fowler PM, Knez W, Crowcroft S, et al. Greater effect of east vs west travel on jet-lag, sleep and team-sport performance. Med Sci Sports Exerc. 2018;50(2):391. doi:10.1249/MSS.00000000000 01522

2. Fowler P, Duffield R, Vaile J. Effects of simulated domestic and international air travel on sleep, performance, and recovery for team sports. Scand J Med Sci Sports. 2015;25(3):441-451. PubMed ID: 24750359 doi:10.1111/sms.12227

3. Fowler PM, Duffield R, Morrow I, Roach G, Vaile J. Effects of sleep hygiene and artificial bright light interventions on recovery from simulated international air travel. Eur J Appl Physiol. 2015;115(3): 541-553. PubMed ID: 25387805 doi:10.1007/s00421-014-3043-2

4. Reilly T, Waterhouse J, Edwards B. Jet lag and air travel: implications for performance. Clin J Sport Med. 2005;24(2):367-380. doi:10. 1016/j.csm.2004.12.004

5. Leatherwood WE, Dragoo JL. Effect of airline travel on performance: a review of the literature. Br J Sports Med. 2012;47(9):561-567. PubMed ID: 23143931 doi:10.1136/bjsports-2012-091449

6. Forbes-Robertson S, Dudley E, Vadgama P, Cook C, Drawer S, Kilduff L. Circadian disruption and remedial interventions: effects and interventions for jet lag for athletic peak performance. Sports Med. 2012;42(3):185-208. PubMed ID: 22299812 doi:10.2165/ 11596850-000000000-00000

7. Sargent C, Ferguson SA, Darwent D, Kennaway DJ, Roach GD. The influence of circadian phase and prior wake on neuromuscular function. Chronobiol Int. 2010;27(5):911-921. PubMed ID: 20636205 doi:10.3109/07420528.2010.488901

8. Reilly T, Atkinson G, Budgett R. Effect of low-dose temazepam on physiological variables and performance tests following a westerly flight across five time zones. Int J Sports Med. 2001;22(3):166-174. PubMed ID: 11354518 doi:10.1055/s-2001-16379

9. Williams B, Clarke R, Aspe R, Cole M, Hughes J. Managing performance throughout periods of travel. Strength Cond J. 2017; 39(4):22-29. doi:10.1519/SSC.0000000000000317

10. Paul MA, Gray GW, Lieberman HR, et al. Phase advance with separate and combined melatonin and light treatment. Psychopharmacology. 2011;214(2):515-523. PubMed ID: 21069516 doi:10.1007/ s00213-010-2059-5

11. Atkinson G, Buckley P, Edwards B, Reilly T, Waterhouse J. Are there hangover-effects on physical performance when melatonin is ingested by athletes before nocturnal sleep? Int J Sports Med. 2001;22(3):232234. PubMed ID: 11354528 doi:10.1055/s-2001-16387

12. Atkinson G, Jones H, Edwards BJ, Waterhouse JM. Effects of daytime ingestion of melatonin on short-term athletic performance. Ergonomics. 2005;48(11-14):1512-1522. PubMed ID: 16338717 doi:10.1080/00140130500100967

13. Thompson A, Batterham AM, Jones H, Gregson W, Scott D, Atkinson G. The practicality and effectiveness of supplementary bright light for reducing jet-lag in elite female athletes. Int J Sports Med. 2013;34(7): 582-589. PubMed ID: 23258609

14. Nédélec M, Halson S, Delecroix B, Abaidia A-E, Ahmaidi S, Dupont G. Sleep hygiene and recovery strategies in elite soccer players. Sports Med. 2015;45(11):1547-1559. doi:10.1007/s40279015-0377-9

15. Semenick D. Testing protocols and procedures. In: Baechle TR, Earle RW, eds, Essentials of Strength Training and Conditioning. Champaign, IL: Human Kinetics; 1994:258-273.

16. Galambos SA, Terry PC, Moyle GM, Locke SA, Lane AM. Psychological predictors of injury among elite athletes. Br J Sports Med. 2010: 39:351-354. 
17. Roach GD, Sargent C. Interventions to minimise jet lag after westward and eastward flight. Front Physiol. 2019;10:927. PubMed ID: 31417411 doi:10.3389/fphys.2019.00927

18. Czeisler CA, Kronauer RE, Allan JS, et al. Bright light induction of strong (type 0) resetting of the human circadian pacemaker. Science. 1989;244(4910):1328-1333. PubMed ID: 2734611 doi:10.1126/ science. 2734611

19. Eastman CI, Burgess HJ. How to travel the world without jet lag. Sleep Med Clin. 2009;4(2):241-255. PubMed ID: 20204161 doi:10. 1016/j.jsmc.2009.02.006

20. Vandenbogaerde TJ, Hopkins WG. Monitoring acute effects on athletic performance with mixed linear modeling. Med Sci Sports Exerc. 2010;42(7):1339-1344. PubMed ID: 20068494 doi:10.1249/ MSS.0b013e $3181 \mathrm{cf} 7 \mathrm{f} 3 \mathrm{f}$

21. Hopkins WG, Marshall SW, Batterham AM, Hanin J. Progressive statistics for studies in sports medicine and exercise science. Med Sci Sports Exerc. 2009;41(1):3-13. PubMed ID: 19092709 doi:10.1249/ MSS.0b013e31818cb278

22. Batterham AM, Hopkins WG. Making meaningful inferences about magnitudes. Int J Sports Physiol Perform. 2006;1(1):50-57. PubMed ID: 19114737 doi:10.1123/ijspp.1.1.50

23. Waterhouse J, Edwards B, Nevill A, et al. Do subjective symptoms predict our perception of jet-lag? Ergonomics. 2000;43(10), 15141527. PubMed ID: 11083132 doi:10.1080/001401300750003943
24. Reilly T, Atkinson G, Edwards B, et al. Coping with jet-lag: a position statement for the European College of Sport Science. Eur J Sport Sci. 2007;7(1):1-7. doi:10.1080/17461390701216823

25. Atkinson G, Batterham AM, Dowdall N, Thompson A, van Drongelen A. From animal cage to aircraft cabin: an overview of evidence translation in jet lag research. Eur J Appl Physiol. 2014; 114(12):2459-2468. PubMed ID: 25342081 doi:10.1007/s00421-0143026-3

26. Reilly T, Piercy M. The effect of partial sleep deprivation on weightlifting performance. Ergonomics. 1994;37(1):107-115. PubMed ID: 8112265 doi:10.1080/00140139408963628

27. Reilly T, Waterhouse J, Edwards B. Some chronobiological and physiological problems associated with long-distance journeys. Travel Med Infect Dis. 2009;7(2):88-101. PubMed ID: 19237142 doi:10.1016/j.tmaid.2008.05.002

28. Waterhouse J, Edwards B, Nevill A, et al. Identifying some determinants of "jet lag" and its symptoms: a study of athletes and other travellers. Br J Sports Med. 2002;36(1):54-60. PubMed ID: 11867494 doi:10.1136/bjsm.36.1.54

29. Skein M, Duffield R, Edge J, Short MJ, Mundel T. Intermittent-sprint performance and muscle glycogen after $30 \mathrm{~h}$ of sleep deprivation. Med Sci Sports Exerc. 2011;43(7):1301-1311. PubMed ID: 21200339 doi:10.1249/MSS.0b013e31820abc5a 